\title{
Validation of two-dimensional vertebral body parameters in estimating patient height in elderly patients
}

\author{
1,2 MOHAMMED ABDUL WADUUD, MRCS MSC, 'PENELOPE POPPY JANPEN SUCHARITKUL, \\ ${ }^{2}$ MICHAEL DROZD, MBChB BSC, ${ }^{1}$ ANKIT GUPTA, ${ }^{3}$ CHRISTOPHER HAMMOND, FRCR MRCS MA, \\ ${ }^{1,2}$ DAVID JULIAN ASHBRIDGE SCOTT, FRCS MD and On behalf of Vascular Surgeons and Interventional Radiologists \\ at the Leeds Vascular Institute \\ ${ }^{1}$ The Leeds Vascular Institute, Leeds General Infirmary, Leeds Teaching Hospital NHS Trust, Great George St, Leeds LS1 3EX, United \\ Kingdom \\ ${ }^{2}$ Leeds Institute for Cardiovascular and Metabolic Medicine, University of Leeds, LIGHT Laboratories, Level 7, Leeds, LS2 9JT, United \\ Kingdom
}

${ }^{3}$ Department of Radiology, Leeds General Infirmary, Leeds Teaching Hospital NHS Trust, Great George St, Leeds LS1 3EX, United Kingdom

Address correspondence to: Dr Mohammed Abdul Waduud

E-mail:m.a.waduud@leeds.ac.uk

Objectives Standardised comparison of abdominal muscle and adipose tissue is often utilised in morphometric clinical research. Whilst measurements are traditionally standardised against the patient's height, this may not be always practically feasible. The aim of this study was to investigate the relationship between measurements of the vertebral body and patient height.

Methods We analysed cross-sectional CT scans. Measurements of the vertebral body area (VBA), anteroposterior vertebral body diameter (APVBD) and lateral vertebral body diameter (LVBD) were made by two independent investigators by manual tracing. Patients were randomly divided into two groups: Group 1 standardisation and Group 2 validation. We compared height and vertebral body parameters from patients in Group 1 and mathematically modelled this relationship. We then utilised the model to predict the height of patients in Group 2 and compared this with their actual height.
Observer variability was assessed using Bland-Altman plots and $t$-tests of differences.

Results CT scans from 382 patients were analysed. No significant intraobserver or interobserver differences were apparent when measuring vertebral body parameters. We describe models which enable the prediction of the patients' height using the measured VBA, APVBD and LVBD. No significant differences were observed between the patients predicted and actual heights in the validation group.

Conclusions We demonstrate an important relationship between measurements of the patient's height and the vertebral body. This can be utilised in future research when the patient's height has not been measured.

Advances in knowledge In the absence of the patient's height, we demonstrate that two-dimensional vertebral body parameters may be reliably used to standardise morphometric measurements.

\section{INTRODUCTION}

Single slice cross-sectional CT and MRI at the level of third lumbar vertebrae or umbilicus are routinely used in clinical research to make morphometric measurements for clinical risk stratification. ${ }^{1-3}$ Both sarcopaenia and abdominal obesity, notably visceral fat, have been shown to be associated with worse patient outcomes. ${ }^{1-3}$ However, measurements of the abdominal muscle and adipose tissue area are commonly standardised against the height of the patient, in a similar manner to the BMI. ${ }^{1,4,5}$ When studies have failed to standardise measurements, they have often been criticised. $^{6}$

In certain circumstances, it may not be possible to measure patient height due to physical limitations, acute illness or lack of equipment. Furthermore, height is not always recorded in the hospital records and this poses a substantial challenge for stratification methods which utilise this basic parameter. Furthermore, biological profiling of human tissue is an essential component of forensic medicine. Despite well-established methods of estimating patient height from specimens such as measuring the size and shape of the cranium, pelvis and long bones, this may not be possible due to missing skeletal material. ${ }^{7,8}$ 
Figure 1. Illustration of cross-sectional CT analysis for measurement of the vertebral body. A (yellow line): demarcates the measured VBA. B (line): demarcates the APVBD. C (red line): demarcates the LVBD.

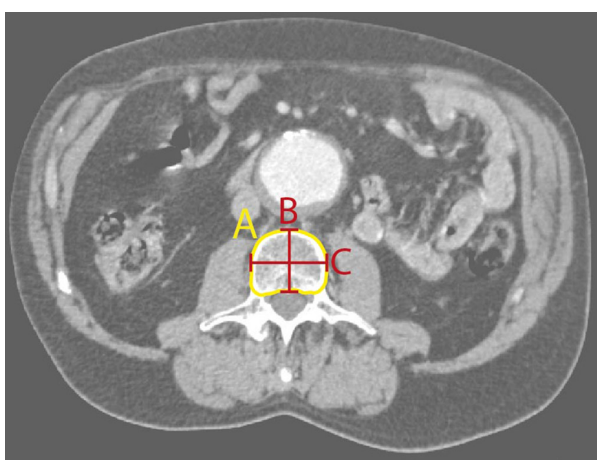

Measurements of the vertebral parameters such as the height, anteroposterior diameter, lateral diameter and vertebral body area (VBA) at different levels of the vertebrae have been previously shown to correlate with patient height. ${ }^{9-12}$ However, these studies have been criticised for having small sample sizes and large margins of error in estimating the height.

The validation of VBA as a surrogate measure of height would offer a simple alternative measure where conventional methods are not feasible. Given the similarities previously observed, we hypothesised that similar relationships may exist between patient height and the VBA at the upper end-plate of the third lumbar vertebrae which is the most commonly used cross-section in radiological morphometric clinical research. ${ }^{1}$ The two measures may have a relationship which could be used to interchangeably standardise morphometric measurements. The aim of this study was to evaluate the relationship between cross-sectional measurements of the vertebral body at the third lumbar vertebrae and patient's height.

Figure 2. Flow chart illustrating the inclusion and exclusion of local patients from the NVR. AAA, abdominal aortic aneurysm; NVR, National Vascular Registry.

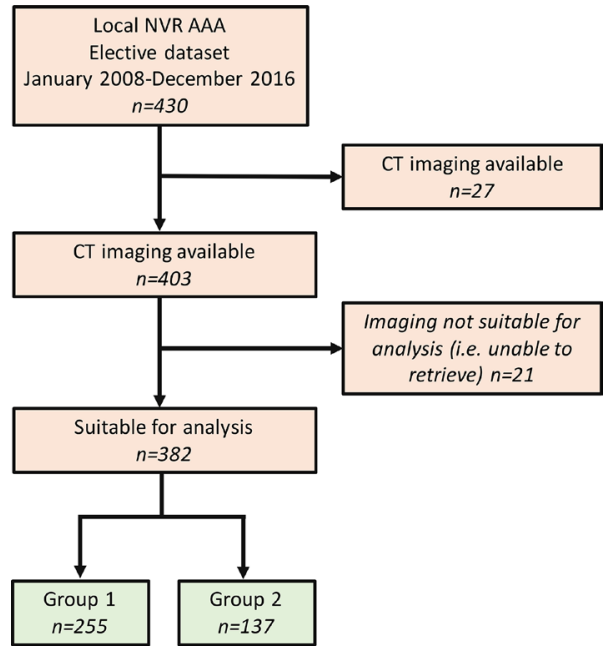

\section{METHODS}

We analysed preoperative cross-sectional CT abdominal imaging from patients who had undergone abdominal aortic aneurysm (AAA) intervention which included endovascular aneurysm repair (EVAR) and open surgical repair (OSR). Consecutive patients were identified from a prospectively national database, the Health Quality Improvement Partnership National Vascular Registry (NVR) between January 2008 and December 2016. ${ }^{13}$ Patients were included if they had undergone a documented height in the patient records and a cross-sectional CT image available at the level of the third lumbar vertebrae. Patients were excluded from the study if portions of the imaging were missing. All data were anonymised prior to analysis.

Preoperative CT imaging of the abdomen is routinely performed on all patients undergoing planned elective intervention for AAA. Scans were performed using a Siemens Somatom Definition AS CT scanner (Siemens Healthcare, Forchheim, Germany) with the patient supine, with a breath-hold to minimise motion artefact. All imaging was performed solely for a preoperative planning using routine CT settings.

Ethical approval was granted by the local radiology research authorisation group and Health Research Authority.

\section{Data collection and image analysis}

Demographic data collected included the patients age, gender, height and weight. Patient height was measured using a stadiometer to the nearest $0.1 \mathrm{~cm}$ by a trained healthcare professional, as previously described. ${ }^{14,15}$ These parameters are routinely recorded in the NVR, however, have only been mandatory fields since January 2013. Case notes were subsequently reviewed to refine the data retrieved.

Images were reviewed on the picture archiving and communications system viewer IMPAX (AGFA-Gevaert Group, Mortsel, Belgium) described briefly in Figure 1. All images were assessed for inclusion by a single investigator who did not participate in

Figure 3. Bland-Altman plots with limiting of agreement illustrating no significant intraobserver and interobserver differences in measurements of VBA, APVBD and LVBD.
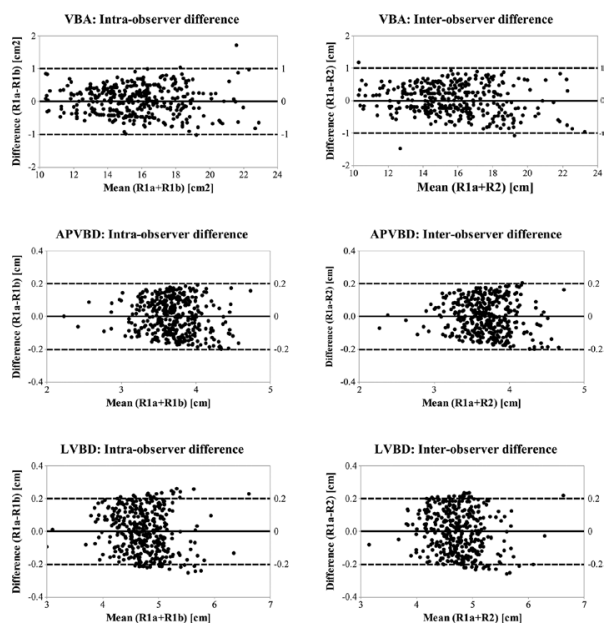
Table 1. Baseline characteristics of two groups used for modelling and validation.

\begin{tabular}{|c|c|c|c|}
\hline Characteristic & $\begin{array}{c}\text { Group } 1 \\
\text { (Mathematical modelling } \\
\text { cohort) } \\
{[N=255]}\end{array}$ & $\begin{array}{c}\text { Group } 2 \\
\text { (Validation cohort) } \\
{[N=127]}\end{array}$ & $p$-value \\
\hline $\begin{array}{l}\text { Age (years) } \\
{[\text { mean } \pm \text { SD }]}\end{array}$ & $75.4 \pm 7.8$ & $74.3 \pm 7.0$ & 0.153 \\
\hline $\begin{array}{l}\text { Height }(\mathrm{cm}) \\
{[\text { mean } \pm \mathrm{SD}]}\end{array}$ & $173.2 \pm 7.8$ & $174.3 \pm 8.7$ & 0.234 \\
\hline $\begin{array}{l}\text { Weight }(\mathrm{kg}) \\
{[\text { mean } \pm \mathrm{SD}]}\end{array}$ & $81.9 \pm 17.9$ & $84.4 \pm 20.2$ & 0.297 \\
\hline \multicolumn{4}{|c|}{ Indication for imaging } \\
\hline $\begin{array}{l}- \text { OSR } \\
{[\mathrm{n}, \%]}\end{array}$ & $87(34.1 \%)$ & $42(33.1 \%)$ & 0.712 \\
\hline \multicolumn{4}{|c|}{ Vertebral body measurements } \\
\hline $\begin{array}{l}-\mathrm{VBA}\left(\mathrm{cm}^{2}\right) \\
{[\text { mean } \pm \mathrm{SD}]}\end{array}$ & $15.7 \pm 2.5$ & $15.9 \pm 2.6$ & 0.395 \\
\hline $\begin{array}{l}-\operatorname{APVBD}(\mathrm{cm}) \\
{[\text { mean } \pm \mathrm{SD}]}\end{array}$ & $3.7 \pm 0.3$ & $3.7 \pm 0.4$ & 0.774 \\
\hline $\begin{array}{l}-\operatorname{LVBD}(\mathrm{cm}) \\
{[\text { mean } \pm \mathrm{SD}]}\end{array}$ & $4.7 \pm 0.4$ & $4.7 \pm 0.4$ & 0.418 \\
\hline
\end{tabular}

the analysis of images. Transverse cross-sectional images were identified by counting up from the sacrum to the third lumbar vertebrae on the sagittal view of the abdomen on multiplanar reconstruction.

The VBA was manually traced at the upper end-plate of the third lumbar vertebrae. The anteroposterior vertebral body diameter (APVBD) and lateral vertebral body diameter (LVBD) were also recorded. Two independent investigators (Rater1 [R1a] and Rater2 [R2]) were trained to analyse images by a senior investigator with prior experience with this technique. Following an initial round of analyses, Rater1 re-analysed all images (R1b) presented in a random order blinded to their previous results, therefore all images were analysed by R1 twice.

\section{Statistical analysis}

Areas were measured in centimetres-squared $\left(\mathrm{cm}^{2}\right)$. Linear measurements were reported in centimetres $(\mathrm{cm})$. Continuous variables were checked for normality and reported as a mean and standard deviation (SD), and were compared using t-tests. Categorical variables are reported as absolute numbers (n) and percentages (\%), and were compared using the chi-square test.

Intraobserver and interobserver differences in measuring VBA, APVBD and LVBD were evaluated using Bland-Altman plots and paired t-tests to ensure that measurements were reproducible. The limits of agreement are illustrated as two SD from the mean differences observed. Measurements from R1a were solely used to formulate a mathematical algorithm that could predict the patient's height, as single observer measurements would be a reflection of the likely clinical utilisation.

The study cohort was subsequently randomly divided into Group 1 (mathematical modelling cohort) and Group 2 (validation cohort) in a 2 to 1 ratio, respectively, using an online random allocator ( random.org). Measurements from Group1 were used to identify the relationship between height and vertebral body measurements using Pearson's correlation. Any significant correlations observed were modelled using linear regression analysis which were adjusted for age and gender. The model formulated could be used to predict the height given a vertebral body parameter. Validation of the model was undertaken using Group 2. Values of the actual and predicted height were compared using Bland-Altman plots and paired $t$-tests. $P$-values less than 0.05 were considered significant. All statistical analyses were performed using Minitab 17 (Minitab Inc., Pennsylvania, USA).

\section{RESULTS}

\section{Patient characteristics}

The study population has previously been described. ${ }^{16}$ In brief, 382 patients had imaging suitable for analysis (Figure 2): 253 (66.2\%) EVAR and 129 (33.8\%) OSR. The mean age of the study population was $75.0 \pm 7.6$ years and $333(87.2 \%)$ were males. The mean height was $174.0 \pm 8.4 \mathrm{~cm}$.

\section{Intraobserver and interobserver differences}

There were no significant intraobserver differences when measuring the VBA (mean difference $0.0 \pm 0.5 \mathrm{~cm}^{2}, p=0.343$ ), 
Figure 4. Linear regression modelling illustrating significant positive relationships between height and vertebral body parameters.
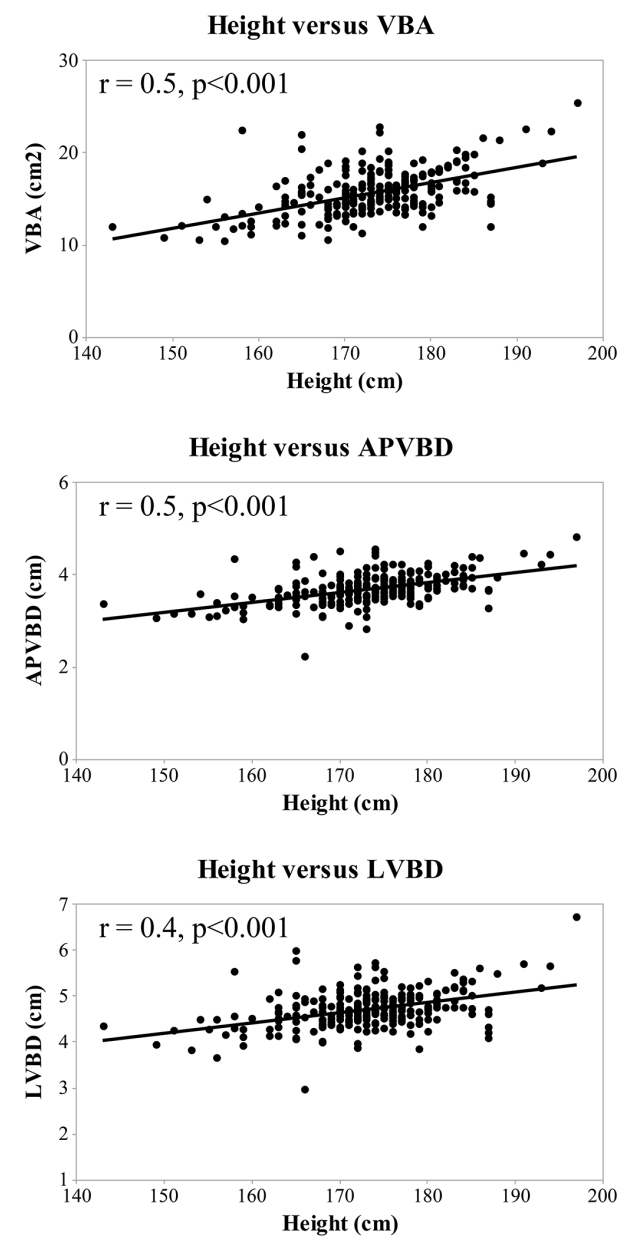

APVBD (mean difference $0.0 \pm 0.1 \mathrm{~cm}, p=0.950$ ) and LVBD (mean difference $0.0 \pm 0.1 \mathrm{~cm}, p=0.976$ ). There were no significant differences in interobserver measurements: VBA (mean difference $0.0 \pm 0.5 \mathrm{~cm}^{2}, p=0.416$ ), APVBD (mean difference 0.0 $\pm 0.1 \mathrm{~cm}, p=0.986$ ) and LVBD (mean difference $0.0 \pm 0.1 \mathrm{~cm}, p=$ 0.941 ). Differences are illustrated in Figure 3. The mean VBA was $15.7 \pm 2.5 \mathrm{~cm}^{2}$. The mean APVBD and LVBD were $3.7 \pm 0.3 \mathrm{~cm}$ and $4.7 \pm 0.4 \mathrm{~cm}$, respectively.

\section{Mathematical modelling and validation}

There were 255 patients (66.8\%) in Group 1 and 127 patients (33.2\%) in Group 2. The two groups had similar baseline characteristics with no significant differences indicating satisfactory randomisation (Table 1).

Comparisons between height and the vertebral body parameters from patients in Group 1 are illustrated in Figure 4. Significant positive correlations were observed between height and all vertebral body parameters (Figure 4). Furthermore, these relationships were quantified by linear regression modelling (Table 2). Patient height may be estimated using the following equations:
$[$ A $]$ Estimated height $=157.4+[$ VBA $\times 1.3]-[$ Age $\times 0.1]+6.7[$ if male $]$
$[$ B $]$ Estimated height $=143.1+[$ APVBD $\times 9.1]-[$ Age $\times 0.1]+6.8[$ if male $]$
$[\mathrm{C}]$ Estimated height $=149.2+[\mathrm{LVBD} \times 5.7]-[$ Age $\times 0.1]+7.9[$ if male $]$

These equations enabled the prediction of the patient's height in the hypothetical absence of a documented height in Group 2. No significant differences were observed on paired $t$-tests comparing actual heights to predicted heights from the VBA, APVBD and LVBD (Table 3). Differences are illustrated in Figure 5.

\section{DISCUSSION}

In this study, we validated the use of vertebral body parameters as a plausible alternative to the patient's height when standardising morphometric measurements from two-dimensional transverse cross-sectional CT imaging. We illustrate the patients height to correlate with the patients VBA, APVBD and LVBD at the third lumbar vertebrae. Our data adds the growing body of evidence describing this relationship. ${ }^{9-11}$ It is feasible that future studies may utilise these parameters to standardise single slice radiological measures of sarcopaenia and obesity, in the absence of anthropometric clinical data. However, we would advocate the use of the patient's height, if available, due to the error associated with using these surrogate derivatives as demonstrated by the wide limits of agreement when comparing actual and predicted heights.

It is important to acknowledge that searching through case notes for large datasets is extremely labour intensive and locating the notes may be challenging as they may be stored over multiple storage sites, in transit or in clinical use. This partly highlights some of the problems of standardising measures to patient height, particularly for clinical research. Despite patient height being a field in the NVR, it has only been made a mandatory field requiring entry since $2013 .{ }^{13}$ Nevertheless, an increasing number of health boards within the National Health Service are migrating

Table 2. Linear regression modelling of height in relation to VBA, APVBD and LVBD

\begin{tabular}{|l|c|c|c|c|c|c|}
\hline Vertebral body parameter & Regression coefficient & R2 & p-value & $\begin{array}{c}\text { Adjusted } \\
\text { Regression coefficient }^{\mathrm{a}}\end{array}$ & R2 & $\boldsymbol{p}_{\text {-value }}$ \\
\hline VBA & 1.6 & 0.3 & $<0.001$ & 1.3 & 0.4 & $<0.001$ \\
\hline APVBD & 11.7 & 0.3 & $<0.001$ & 9.1 & 0.4 & $<0.001$ \\
\hline LVBD & 7.6 & 0.2 & $<0.001$ & 5.7 & $<0.3$ & $<0.001$ \\
\hline
\end{tabular}

aanalysis was adjusted for age and gender. 
Table 3. Observed differences in estimated height and actual height using vertebral parameters

\begin{tabular}{|l|c|c|c|}
\hline & $\begin{array}{c}\text { Estimated height } \\
{[\text { mean } \pm \text { SD] }}\end{array}$ & $\begin{array}{c}\text { Difference from actual height } \\
{[\text { mean } \pm \text { SD] }}\end{array}$ & $p$-value \\
\hline VBA & $173.7 \pm 5.0$ & $0.6 \pm 6.3$ & 0.276 \\
\hline APVBD & $173.5 \pm 5.0$ & $0.8 \pm 6.6$ & 0.164 \\
\hline LVBD & $173.6 \pm 4.4$ & $0.7 \pm 6.6$ & 0.222 \\
\hline
\end{tabular}

${ }^{a}$ paired $t$-test between predicted and actual documented patient height.

Figure 5. Bland-Altman plots with limiting of agreement illustrating no significant differences in the actual and predicted height.
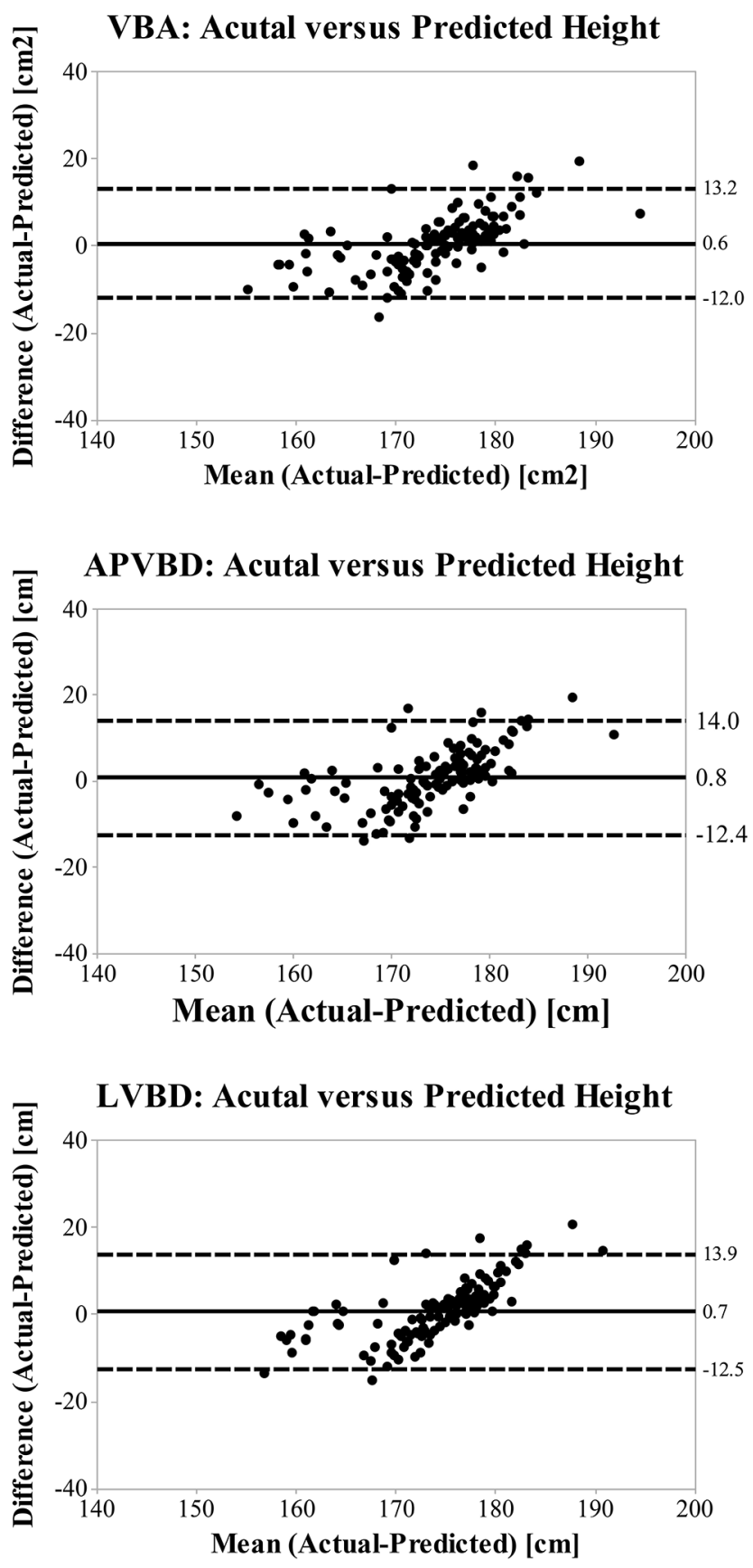

over to digital platforms, which should make the retrieval of the patient's height relatively simple and facilitate the standardisation of results to the patients height in future retrospective studies. ${ }^{17,18}$ Relaying solely on this strategy may, however, limit future prospective study outcomes to short-term outcomes as these systems have only recently begun to be implemented.

Vertebral body dimensions may be measured in a reliable and highly reproducible method independent of observer bias. We have previously shown that it is possible to make such measurements with minimal training without the need for expert review by a radiologist. ${ }^{19}$ Furthermore, this method may be utilised at the same time as measuring the abdominal muscle or adipose tissue in order to standardise measurements. Measurements of the vertebral body dimensions may be converted to the predicted height, using the formulae described. However, we acknowledge that in such scenarios the VBA is estimating the patient's height and comparisons should be exercised with caution. We report a similar SD in estimating patient height to that recently described by Oura et al. ${ }^{11}$ Furthermore, we did not assess the vertebral column for the presence of wedge fractures which may influence patient height, nor did we identify the presence of osteophytes which may influence VBA measurement. However, neither of these factors appeared to affect the relationship observed between patient height and the VBA.

We intentionally selected only elective patients in this study as patients were more likely to have a preoperative height documented in combination with preoperative CT imaging for operative planning. However, a large proportion of patients who had elective intervention at our centre did not have a documented height, which we were unable to retrieve despite searching through patient case notes. We split the study population into unequal cohorts so that the regression model would be formulated from a larger patient sample therefore more likely to be representative of the patient population. Furthermore, we measured the VBA at the third lumbar vertebrae instead of the fourth lumbar vertebrae, as previously evaluated by Oura et al, as we have previously shown morphometric measurements at this level to be most representative of structural volume. ${ }^{20}$ Despite this, the margin of error in estimating patient height was comparable. Oura et al demonstrated volumetric quantification of the VBA to provide the most accurate representation of patient height. This was beyond the scope of this study as we focused on simple two-dimensional measurement of vertebral parameters which could be performed at the same time as making other simple morphometric characterisation of fat and skeletal muscle. ${ }^{11}$ 
In conclusion, we demonstrate an important relationship between measurements of the patient's height and VBA. We have mathematically modelled and validated this relationship. In the absence of patient height, we have demonstrated that the VBA, APVBD and LVBD may be reliably used to standardise measurements of muscle and adipose tissue area as an alternative to patient height in clinical research.

\section{ACKNOWLEDGMENT}

We would like to thank Mr David Watson in helping us retrieve data from the national vascular registry.

\section{FUNDING}

This research received no specific grant from any funding agency in the public, commercial, or not-for-profit sectors. MAW and MD are clinical research training fellows supported by the British Heart Foundation.

\section{PATIENT CONSENT}

The study was approved by the National Health Service (NHS, UK) Health Research Authority. IRAS project ID 228484.

\section{REFERENCES}

1. Jones K, Gordon-Weeks A, Coleman C, Silva M. Radiologically determined sarcopenia predicts morbidity and mortality following abdominal surgery: a systematic review and meta-analysis. World J Surg 2017; 41: 2266-79. doi: https://doi.org/10.1007/ s00268-017-3999-2

2. Marques MD, Santos RD, Parga JR, Rocha-Filho JA, Quaglia LA, Miname $\mathrm{MH}$, et al. Relation between visceral fat and coronary artery disease evaluated by multidetector computed tomography. Atherosclerosis 2010; 209: 481-6. doi: https:// doi.org/10.1016/j.atherosclerosis.2009.10.023

3. Fox CS, Massaro JM, Hoffmann U, Pou KM, Maurovich-Horvat P, Liu C-Y, et al. Abdominal visceral and subcutaneous adipose tissue compartments. Circulation 2007; 116: 39-48. doi: https://doi.org/10. 1161/CIRCULATIONAHA.106.675355

4. Nicklas BJ, Penninx BWJH, Ryan AS, Berman DM, Lynch NA, Dennis KE. Visceral adipose tissue cutoffs associated with metabolic risk factors for coronary heart disease in women. Diabetes Care 2003; 26: 1413-20. doi: https://doi.org/10.2337/ diacare.26.5.1413

5. Yumi M, Toru N, Shuichiro Y, Yoshihiko T, Tetsuji Y, Tetsuya M, et al. Visceral fat area cutoff for the detection of multiple risk factors of metabolic syndrome in Japanese: the Hitachi health study. Obesity 2012; 20: 1744-9. doi: https://doi.org/10.1038/oby. 2011.285

6. Jones AD, Waduud MA. Re. "Effect of low skeletal muscle mass on post-operative survival of patients with abdominal aortic aneurysm: a prognostic factor review and meta-analysis of time to event data". Eur J Vasc Endovasc Surg 2019; S1078-5884:
313929-9. doi: https://doi.org/10.1016/j.ejvs. 2019.07.037

7. Krishan K, Chatterjee PM, Kanchan T, Kaur S, Baryah N, Singh RK. A review of sex estimation techniques during examination of skeletal remains in forensic anthropology casework. Forensic Sci Int 2016; 261: 165. e1-165.e8. doi: https://doi.org/10.1016/j. forsciint.2016.02.007

8. Trotter M, Gleser GC. A re-evaluation of estimation of stature based on measurements of stature taken during life and of long bones after death. Am J Phys Anthropol 1958; 16: 79-123. doi: https://doi.org/10.1002/ajpa. 1330160106

9. Pelin C, Duyar I, Kayahan EM, Zağyapan R, Ağildere AM, Erar A. Body height estimation based on dimensions of sacral and coccygeal vertebrae. J Forensic Sci 2005; 50: 294-7. doi: https://doi.org/10.1520/ JFS2004010

10. Twomey L, Taylor J. Age changes in the lumbar spinal and intervertebral canals. Paraplegia 1988; 26: 238-49. doi: https://doi. org/10.1038/sc.1988.37

11. Oura P, Korpinen N, Niinimäki J, Karppinen J, Niskanen M, Junno J-A. Estimation of stature from dimensions of the fourth lumbar vertebra in contemporary middleaged finns. Forensic Sci Int 2018; 292: 71-7. doi: https://doi.org/10.1016/j.forsciint.2018. 09.001

12. Zhang K, Chang Y-feng, Fan F, Deng Z-hua, Chang F, Deng Z. Estimation of stature from radiologic anthropometry of the lumbar vertebral dimensions in Chinese. Leg Med 2015; 17: 483-8. doi: https://doi.org/10.1016/ j.legalmed.2015.10.004

13. Waton S, Johal A, Heikkilä K, Cromwell D, Loftus I. National vascula r Registry: 2015 annual report. The Royal College of Surgeons of England. 2015.

14. Rosenbaum S, Skinner RK, Knight IB, Garrow JS. A survey of heights and weights of adults in Great Britain, 1980. Ann Hum Biol 1985; 12: 115-27. doi: https://doi.org/10. 1080/03014468500007621

15. Voss LD, Bailey BJ, Cumming K, Wilkin TJ, Betts PR. The reliability of height measurement (the Wessex growth study. Arch Dis Child 1990; 65: 1340-4. doi: https:// doi.org/10.1136/adc.65.12.1340

16. Waduud MA, Wood B, Keleabetswe P, Manning J, Linton E, Drozd M, et al. Influence of psoas muscle area on mortality following elective abdominal aortic aneurysm repair. Br J Surg 2019; 106: 367-74. doi: https://doi.org/10.1002/bjs.11074

17. Nicholas D, Huntington P, Williams P, Gunter B. Digital visibility: menu prominence and its impact on use. Case study: the NHS direct digital channel on Kingston interactive television. Aslib Proc 2002; 54: 213-21. doi: https://doi.org/10. 1108/00012530210443311

18. Cross M. Keeping the NHS electronic spine on track. BMJ 2006; 332: 656-8. doi: https:// doi.org/10.1136/bmj.332.7542.656

19. Waduud MA, Drozd M, Linton E, Wood B, Manning J, Bailey MA, et al. Influences of clinical experience in the quantification of morphometric sarcopaenia: a cohort study. Br J Radiol 2018; 091: 20180067. doi: https:// doi.org/10.1259/bjr.20180067

20. Waduud MA, Adusumilli P, Drozd M, Bailey MA, Cuthbert G, Hammond C, et al. Volumetric versus single slice measurements of core abdominal muscle for sarcopenia. $\mathrm{Br}$ J Radiol 2019; 92: 20180434. doi: https://doi. org/10.1259/bjr.20180434 